\title{
Forecasting adverse weather situations in the road network
}

\author{
Vicente R. Tomás, M. Pla-Castells, Member, IEEE, Juan José Martínez and Javier Martínez
}

\begin{abstract}
Weather is an important factor that affects traffic flows and road safety. Adverse weather situations affect the driving conditions directly, so drivers must be informed about the weather conditions downstream in order to adapt their driving. In the frame of ITS, several systems have been developed to know the weather situations and inform drivers. However, these systems do not forecast weather in advance and they need the support of road operators to inform drivers. This paper presents a new autonomous system to forecast weather conditions in a short time and to give users the information obtained. The system uses a set of algorithms and rules to determine the weather and to forecast dangerous situations on the road network. It has been implemented using a multi-agent approach and tested with real data. Results are very promising. The system is able to forecast adverse situations with a high degree of quality. This quality makes it possible to trust in the system and to avoid the supervision of operators.
\end{abstract}

Index Terms-Multiagent system, expert systems, intelligent transport systems.

\section{INTRODUCTION}

W EATHER is an important factor that affects traffic flows and road safety. Adverse weather conditions have a big impact on accident rates. In [1], the Department of Transport of US presented a study of the impact of weather on roads. The analysis shows that $24 \%$ of the accidents are related to adverse weather conditions. In Spain, $19 \%$ of accidents are produced in adverse weather situations [2]. In Finland, 47\% of fatal crashes occur during the winter period [3].

Adverse weather conditions affect directly driving conditions. Fog affects visibility and the capacity of the driver to see other cars, snow and rain reduces visibility but also increases the risk of slippery roads and wind speed affects the stability of the vehicle.

As many authors have studied before, weather conditions modify the basis of traffic flows [4], [5]; road capacity decreases $(\mathrm{Veh} / \mathrm{h})$, occupancy increases (veh/lane), braking distance increases [6] and even the drivers' response times are higher [7]. However, previous studies [8] reveal that drivers do not adapt their driving to adverse weather conditions. For instance, the headway differentials with dry or wet surface are almost the same, and the average speed is not reduced significantly. This mismatch between driving environments and the actual driving behaviour leads to an increasing risk of

Vicente R. Tomás is with the Department of Computer Science and Engineering, Universitat Jaume I. Av. de Vicent Sos Baynat, s/n (12071) Castellón de la Plana, Castellón. Spain e-mail: (vtomas@uji.es).

M. Pla-Castells, Juan José Martínez and Javier Martínez are with IRTIC Universitat de Valencia Catedrático José Beltran, 2, (46980) Paterna, Valencia. Spain email: (Marta.Pla@uv.es,juanjo@irtic.uv.es,Javier.MartinezPlume@uv.es)

Manuscript received xxxx, xxx; revised September xxxx, xxxxx. crashes, so weather information systems are needed to warn drivers about the driving conditions.

Road safety could be improved providing real-time local information to the road user, especially during the first period of the adverse situation [9]. Currently, one of the most used on-trip information systems is Variable Message Signs (VMS). VMS are located on the road and connected to the Traffic Control Centre (TCC) that can display different messages in the sign. Depending on the type of VMS, messages can be: text, pictogram or both.

Traffic management in real time is a complex task since it presents a high level of distribution. Roads have a huge extension and different equipments are used to monitor the traffic status. Therefore, road traffic managers need support to control and manage the traffic situation. These systems are known as Advanced Traffic Management Systems (ATMS). The combined use of the different elements of ATMS, especially weather monitoring (meteorological stations) and information systems (VMS, Radio Data System -Traffic Message Channel RDS-TMC...), could improve road safety in local areas. However, it requires a constant monitoring by road operators. So, to use these systems in a huge road network, a non-supervised system that will support road operators is required.

Furthermore, the weather information provided by public and regional weather report services, as well as being more general and not as accurate as dedicated systems, refer to a wider land and not to a specific point or segment on the road network.

In this domain, an autonomous system is defined as a system able to monitor weather and traffic information, to analyze and process it and to provide relevant information to drivers without the supervision of the TCC. Thus, autonomous systems require confidence not only about the information they provide, but also the information or data they receive. In consequence, data quality is the key element for these kinds of systems [10]. This quality is being analyzed in several projects and organizations [11]-[14]. In these projects, not only the content and processing detection is analyzed but also the importance of service provision.

In this paper we are going to present a new extension of the expert system to identify adverse weather situations in a local road network. The objective of this extension is to provide short-term predictions on adverse weather conditions for the next two hours with a high degree of reliability. So, we improve the system data quality. The improvement of the short-term prediction will increase the system timeliness (time to detect the incident) and the service provision, because no operator is required to warn end-users. 
The paper is structured as follows: In Section II we present a review of traffic information systems to forecast adverse weather situations. In Section III we present a local system to detect and warn about adverse weather situations. In Section IV the proposed system to support road operators and to warn drivers is presented. In Section V we present the results of applying the model to a real case: Spanish A6 motorway weather prediction. Finally, the conclusions and future work are shown in Section VI.

\section{RELATED WORK}

Adverse weather situations (frost, fog, wind, etc.) affect traffic flow. However, these adverse conditions are usually produced at a particular point and it is difficult for regional and national weather services to identify them. Currently, weather information is obtained from the road weather information systems (RWIS) equipment installed nearby the road lane network. This equipment is formed by a set of different sensors that monitors the weather and usually sends the information to the Traffic Control Centre (TCC).

Several systems have been developed to forecast and detect adverse weather situations. In [15], [16] an expert system was designed to provide a 20 hour forecast of roadway and bridge frost. The system is based on the analysis of three meteorological conditions: a) the surface temperature must be below freezing, b) the surface temperature must be below the dew-point temperature and c) the dew-point temperature must be near (in some cases above) freezing. This system does not forecast local road sections but the whole Iowa State (U.S.) using general information provided by weather services rather than a roadside meteo station. It calculates the possible frost areas and calculates the evolution of these areas. The road network and the bridges of the Iowa State have been previously classified in problematic sections and if the frost areas overpass some road or bridge identified as problematic, an alert is fired. The system relies on a dateset of historical data relating to the four previous years.

In [17], the authors have developed a decision support tool to interpret predicted air and dew point temperatures and observed current wind conditions as frost and freeze event warning related to crops for the subsequent 12 -h period. The expert system uses fuzzy rules to determine the event warning alerts. The outputs of the study consider a wider portion of land and don't have to be as specific as those applied to a particular point on the road.

In [18] a model for predicting pavement surface temperature on roads is developed. They use statistical models capable of predicting the pavement surface temperature from the knowledge of weather variables such as air temperature, relative humidity, dew point, precipitation, wind speed, etc. Multivariate regression analysis is used to estimate the pavement temperature as a function of the weather variables. The computational costs of the calculations are too high to consider this method in a real-time prediction system like the system proposed in this paper.

In [19] an expert system to enhance traffic safety was developed. It is based on the road friction coefficient. The system has a slippery condition module, based on rules, to prevent adverse situations (Dry, Wet, Frost and Snow) on the road network. The system presents a similar approach to our work. However, it does not take into account the data quality of the information measured by the sensors of the RWIS. Thus, a high number of false alarms are fired. Moreover, the system is quite static and the rules are predefined, so different combinations of these rules are not possible.

Road surface condition (RSC) and road surface temperature (RST) are two of the main parameters to detect dangerous situations in the road network. The XRWIS system [20] presents an approach to forecast adverse situations based on a spatial forecasting component (route based forecast). It shows a new approach that increases its reliance although it is very expensive as it requires high level of monitoring to determine the RSC and RST along the route. Furthermore the system is focused on road maintenance and not on traffic management. The results of the system are used for determining the routes to be maintained in order to prioritise roads for salt spreaders.

Other models to forecast adverse weather situations are mathematical models that represent the possible evolution of the different atmospheric situations. However, these models have an approximate solution of a system of equations for which no analytical solution is known. This makes the use of numerical models to improve prediction of weather situations necessary. An example of a numerical model is HIRLAM [21].

HIRLAM (HIgh Resolution Limited Area Model) is the result of a project that emerged with the aim of providing a short-term weather prediction with acceptable reliability. It is developed with the cooperation of European meteorological institutes. The programme was initiated in 1985 and has gone through numerous phases till now [22].

HIRLAM gives the probability of occurrence of individual weather events based on a full Numerical Weather Prediction model (NWP) [23]. It includes the assimilation of data with analysis of conventional and unconventional observations, and is capable of restricting the prediction to an area delineated by a parameterization with different physical variables. The prediction is made not only with the version of geospatial statistics or the use of a mesh of points, but also with the spectral version of the model.

The evolution of HIRLAM models shows an important decrease of the errors produced in the forecast process. In a forecast of 2 days, the error is now about half of the value it was in the previous version [24].

However, although it is able to predict individual weather events, HIRLAM presents two main inconveniences to be used as the only detection method in local road traffic weather alerts: the time and location of the forecast. The minimum time of forecast produced by HIRLAM is every 6 hours $(6$ hours, 12 hours and $24 \mathrm{~h}$ ) and this is a long period of time to inform users (drivers). The event location is rather general (up to $16 \mathrm{~km}$ ), so it could affect the road network or not.

Multiagents systems (MAS) present several advantages to develop distributed, intelligent and cooperative systems. These advantages are high flexibility, adaptability, scalability, and robustness in decentralized systems, which are achieved by problem decomposition and the proactive, reactive, and adap- 
tive behavior of intelligent agents. Multiagent systems are mature sophisticated technology to implement autonomous industrial processes [25], [26] and they are also used in route planing [27].

MAS are also used to improve weather forecast. In [28] a comprehensive and truly agent framework has been deployed. This system was an extension of a weather forecast based on a fuzzy neuro system developed before by the same authors. Basically, the new system uses the main characteristics of agents to create an intelligent layer that permits the creation of a MAS platform that is able to gather real-time weather information from different sources and to develop weather predictions.

In [29], a MAS system to coordinate the information provided by environment sensors is developed. The system is based in a meta-level control approach. Each agent is able to operate in two ways: a) deliberate by itself, where it makes decisions to determine the weather and b) mete-level control where it cooperates with other agents to deliberate to make decisions.

Although these two approaches have some elements in common with our approach: agents exchange and combine different data sources to improve the forecast, their approach can not be used in our systems mainly because: a) the forecast is based on historic periods and these periods are greater than the time period available in our system and b) the forecast area is so wide it could not be used to forecast weather at a particular point or segment of the road network with a considerable degree of confidence.

In the frame of Intelligent Transport System (ITS), several MAS systems have been developed and deployed in several areas (traffic monitoring, users information, traffic management...). In [30] a MAS system has been deployed to calculate dynamically the Origin/Destination Matrices using V2I Messages. In [31] a negotiation framework using agent technology has been defined to improve parking in cities. The system is based on a flexible negotiation mechanism that takes into account the preferences of users (not only drivers but also parkings providers) to improve the city parking problem and thus traffic flows. [32] proposes an agent-based framework to optimize the information provided to travelers. The systems combines different users information (where the user is, when the information has to be provided, to whom...) to suggest an alternative route. In [33] a MAS system to improve the traffic light control has been developed. Each agent controls a traffic light intersection and it is able to act in an indirect way (it reacts to dynamic flow when traffic flow is not congested or nearby) and in a direct way (when traffic is near to collapse and the control changes to a green wave based control strategy).

In [34] a new ATMS was exposed to manage efficiently meteorological incidents. The ATMS is based on multiagent and rule-based system technologies. It was designed to work in two modes: locally and coordinated. In the local mode (without active communications with other agents) local agents work autonomously with respect to the local weather information available. In the coordinated mode, local agents work coordinated with other agents in the network to extend the monitored area. They provide weather information and the information to end-users in a long segment or even in different itineraries.

The system presented in this paper is an extension of the meteorological system proposed in [34]. Our new system also presents a distributed approach and it is based on a multiagent and rule-based system technologies. The Marti et al. system is able to forecast and warn users about local road adverse weather situations and our new approach improves the system quality by: a) the extension of the rule system, taking into account the evolution in time of the meteorological variables in order to update the Certainty and b) an improvement of the forecast quality, through the integration of HIRLAM results into the local systems to detect and warn about adverse weather situations.

\section{A LOCAL SYSTEM TO DETECT AND WARN ABOUT ADVERSE WEATHER SITUATIONS}

A Local Environment (LE) is a small group of equipment physically connected. The purpose of this LE is to detect and warn users about adverse weather situations based on the local system. It is based on the system defined in [34]. A LE can work independently of the TCC. The LE monitors meteorological data, analyses it and warns the users in case of meteorological problems. To develop these tasks, the local environment is composed of the following equipment:

- A Road Weather Information System (RWIS) with several weather sensors (visibility, wind speed, precipitations, temperature...).

- A Data Collection Station (DCS), which collects traffic data from the road network (flow, speed, density...).

- One or more Variable Message Signal (VMS), which show messages to road network users.

- A RDS-TMC module, which is a technology for delivering traffic and travel information to drivers via radio signals. (If the local environment does not have any VMS, a RDS-TMC module is required). This way, the local environment is able to warn users via RDS-TMC or VMS.

- A Remote Station (RS). It is the core of the local system. It receives the information from RWIS or DCS and determines what information must be distributed. If there is communication with other local environments or with the TCC it exchanges its information.

The minimum equipment the LE needs to work is at least one VMS and one RS. The system can have any number of VMS. In case it has no VMS, the RS must have a RDSTMC module. If the RS does not have a RDS-TMC module, the system must have at least one VMS. The system can have any number of DCS. Any possible configuration of a LE following these requirements allows the capture and monitoring of meteorological variables, their analysis to detect weather incidents and to inform the users, either by VMS or RDS-TMC. The current I2V interface is made using VMS (directly to the driver) or RDS-TMC (to the on-board vehicle equipment). The weather and traffic information provided by the LE is also sent to the traffic information center when communication is available. This information is codified using the DATEX II standard [35], so other communication I2V services could be easily implemented. The functioning of the 

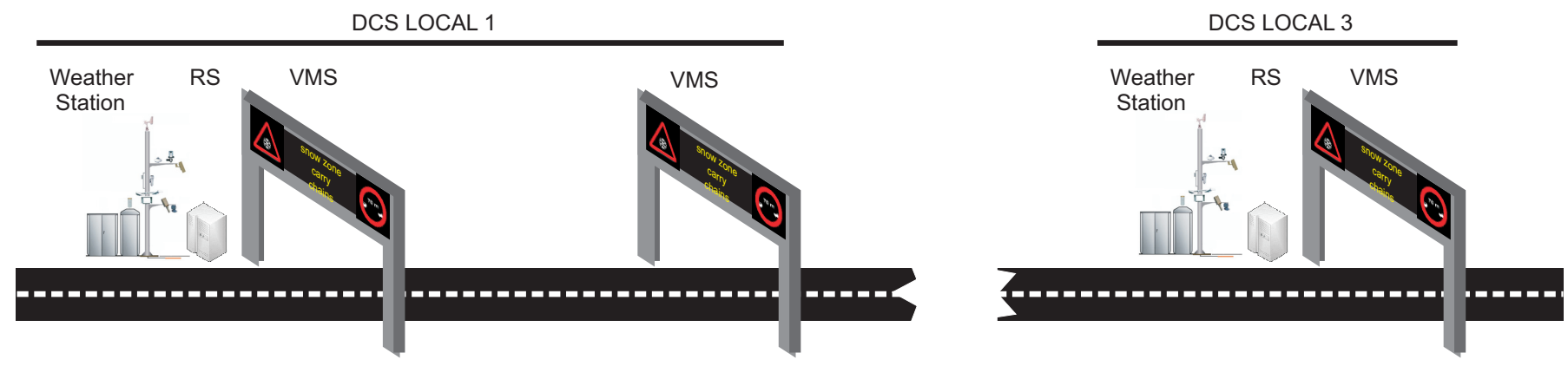

Fig. 1. Example of local areas. Left local area is composed by one RWIS, one RS and two VMS. Right local area is composed by one RWIS, one RS and one VMS

system is the following: the different sensors of RWIS detect weather variations and fires an alarm when a threshold is reached. Then, this information is sent to the RS station. RS analyzes the information applying a set of rules and procedures, and if the alarm set off is taken as correct, it informs users via VMS or RDS-TMC.

The coverage area of the local environment depends on the equipment location, but also on the equipment characteristics itself. For example, if the LE has only VMS, the coverage area is downstream of the VMS location. However, if the LE has a RDS-TMC system, the coverage area is the same as the RDS-TMC influence area.

The main characteristic of the LE is the ability to work autonomously: it forecasts possible dangerous situations based on adverse local weather and it informs drivers via VMS. However, in the local mode, there is no validation from the TCC operator, so the information provided to drivers must be validated to determine its quality before an alarm is fired.

\section{Meteorological variables}

The meteorological variables monitored by the RWIS allow the detection and prognosis of adverse weather conditions. The variables depend on the sensors installed in the RWIS. The most widely used sensors are: Wind Speed, Visibility, Surface State, Height of Snow, Height of the Water Film, Air Relative Humidity, Intensity of Precipitation, Air pressure, Global Radiation, Percentage of Soil Salinity, Soil Freeze Temperature, Air Temperature, Soil surface Temperature, Dew occurrence Temperature and Underground Temperature

Adverse weather conditions can be identified by analysing and combining different meteorological variables. These conditions cause traffic problems that have to be determined and for each of these problems, a set of traffic management measures have to be identified. In the projects Meteosafety [36], EasyWay [12] and EIP [14], the effects of adverse weather problems on traffic were analysed. Table I presents the identified adverse weather conditions for each set of meteorological variables, the associated traffic problems and the different traffic measures to be adopted.

\section{DeTECTION OF LOCAL WEATHER. THE MODEL.}

The identification of adverse weather situations is the key point in our new system. These situations could be identified in two ways:
- An alarm is fired due to the information provided by some sensors, indicating that a certain threshold has been reached.

- The evolution of the different data provided by sensors forecast that an adverse weather situation could be produced.

In both situations data quality is essential. Raw data must be validated in order to guarantee that the traffic management measures adopted are adequate to the current weather situation. Therefore, in the new system we are proposing, before activating an alarm, the RS executes several processes in order to verify the situation. The processes are based on the certainty value and a set of rules: coherence, correlation and prediction (taking also into account historical data). Figure 2 shows the diagram flow of the proposed model for a specific parameter.

a) Detection Algorithm: It monitors, individually, the information received from RWIS sensors and determines the occurrence of a weather incident. The initial occurrence of a weather incident is configured using an initial parameter and several thresholds. For example, visibility values lower than $200 \mathrm{~m}$. fires a fog alarm.

b) Certainty: Each detected meteorological parameter has a value of Certainty associated to it. It establishes the probability of actual occurrence of each situation. It takes a numeric value between 0 (situation not possible) and 100 (completely true situation). The initial value of certainty is determined by the RS following the results of the HIRLAM report. The RS agent has the public internet feed address of the AEMET (Spanish weather agency) and every 6 hours, it checks if there is any new HIRLAM forecast report. If yes, the file is downloaded and it is incorporated into the system.

c) Consistency Algorithm: It analyses the short term evolution of the sensor information individually. It evaluates wether the data that fires the alarm is consistent with the changes measured by the sensor in the last period of time stored locally on the RWIS.

Consistency algorithm increases or decreases the level of certainty of Detection depending on the consistency of data with short-term historical evolution. Furthermore, the Consistency Algorithm could compare the sensor value with the values of the same sensor type in close stations. When the RWIS station executes this algorithm, the agent managing it communicates with the associated RWIS asking for their 


\begin{tabular}{|l|c|c|l|}
\hline PARAMETERS & ADVERSE CONDITION & TRAFFIC PROBLEM & TRAFFIC MEASURE \\
\hline $\begin{array}{l}\text { Intensity of Precipitation } \\
\text { Surface state }\end{array}$ & & & \\
$\begin{array}{l}\text { Height of the water film } \\
\text { Air Relative Humidity } \\
\text { Soil Freeze temperature } \\
\text { Dew occurrence temperature } \\
\text { Percentage of soil salinity } \\
\text { Air Temperature }\end{array}$ & Ice on surface & Slippery & Decrease speed. \\
\hline $\begin{array}{l}\text { Visibility } \\
\text { Intensity of precipitation } \\
\text { Height of the water film } \\
\text { Global radiation }\end{array}$ & Rain & Visibility & Increase distance \\
\hline $\begin{array}{l}\text { Visibility } \\
\text { Global radiation } \\
\text { Air Relative Humidity }\end{array}$ & Slippery & Decrease speed & between vehicles. \\
\hline $\begin{array}{l}\text { Height of snow } \\
\text { Intensity of precipitation } \\
\text { Surface state }\end{array}$ & Fog & Visibility & $\begin{array}{l}\text { Decrease speed. } \\
\text { Increase distance } \\
\text { between vehicles. }\end{array}$ \\
\hline Wind speed & Thow on surface & Slippery & $\begin{array}{l}\text { Decrease speed. } \\
\text { Increase distance } \\
\text { between vehicles. } \\
\text { Chains or Traction } \\
\text { tires required. }\end{array}$ \\
\hline
\end{tabular}

SOME RWIS WEATHER PARAMETERS, THEIR ADVERSE CONDITION AND THE RELATED TRAFFIC PROBLEMS

weather values. The associated RWIS are located within a ten $\mathrm{km}$ radius (this value can be updated depending on the road topography). The communication between RWIS agents is developed using the FIPA-QUERY standard protocol for multiagent communications and interactions [37] [38].

d) Correlation Algorithm: This algorithm correlates the information of different RWIS sensors to assess the quality of the information that fires the alarm. It evaluates the relationship of one variable with other variables. The correlation functions to be applied depend on the meteorological variable that fires the alarm.

e) Forecasting algorithm: This algorithm performs a short-term prediction about the situation that could be produced in a short-term period. It uses the current values of the parameters of the weather stations, validated by coherence and correlation algorithms, to analyse the evolution of some parameters in order to determine and forecast adverse weather situations. These situations are also compared with groups of historical data to establish trends and search for similar situations.

f) Historic algorithm: It uses the RS local database to compare the current situation with previous historical situations. When an alarm is fired by the RS, the information is stored in its own database. If this situation became true (the operator indicated it, in real-time or off-time) not only the individual parameter, but also the related ones are stored.

As an example, Figure 3 shows two different situations to present the operation of the algorithms and the importance of the correlation and coherence algorithms introduced in our method. The top figure (Figure 3(a)), shows the evolution of visibility and intensity of precipitation. The correlation algorithm identifies this situation as feasible (precipitation has influence over visibility) and increases the degree of certainty due to the relation of parameters. The bottom Figure (Figure $3(\mathrm{~b})$ ), presents the temperature parameter evolution. In this example, the coherence algorithm determines that there are some problems, due to the historic evolution: the measured data during the last four hours has no variations, so it is possible that this sensor is not working properly.

\section{A. Description of the whole process}

The main objective of our new system is to identify potential dangerous situations accurately that may affect traffic in an automated way. Every data monitored by the RWIS sensors is integrated into the meteorological stations (MS) in periods of 10 minutes. Previous to the process of integrating this value, it is assessed using the Correlation and Coherence algorithms. If the result of these processes is good, a new Certainty Value is provided and the data is stored in the local database. Then, two processes executed in parallel start: a) the data is analysed by the detection algorithm in order to check the corresponding alarm; and b) the forecast algorithm analyses this new data and compares it with its related meteorological parameters in order to determine if potential dangerous situations are identified. The result of the whole process is compared with the historical values for similar situations in order to increase or decrease the Certainty.

As we mentioned before, when the system forecasts a situation, and later, this situation is confirmed, the certainty is increased and it is stored as a true situation. In any case, the 


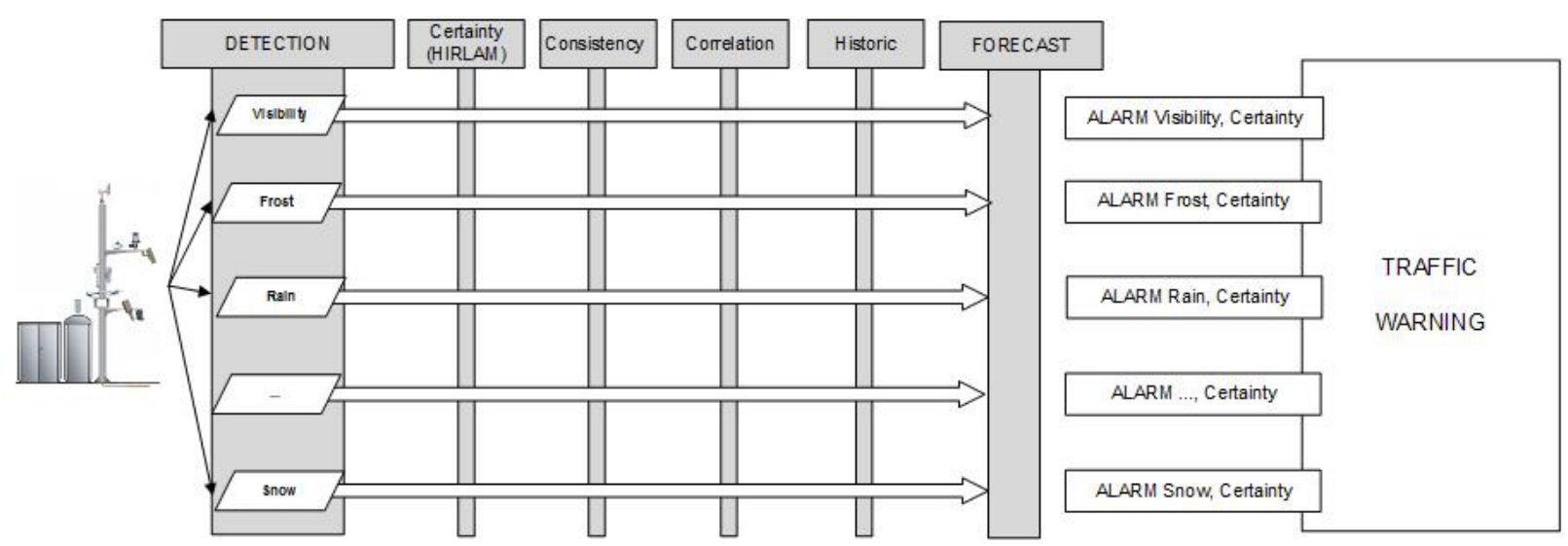

Fig. 2. New model schema for different weather parameter

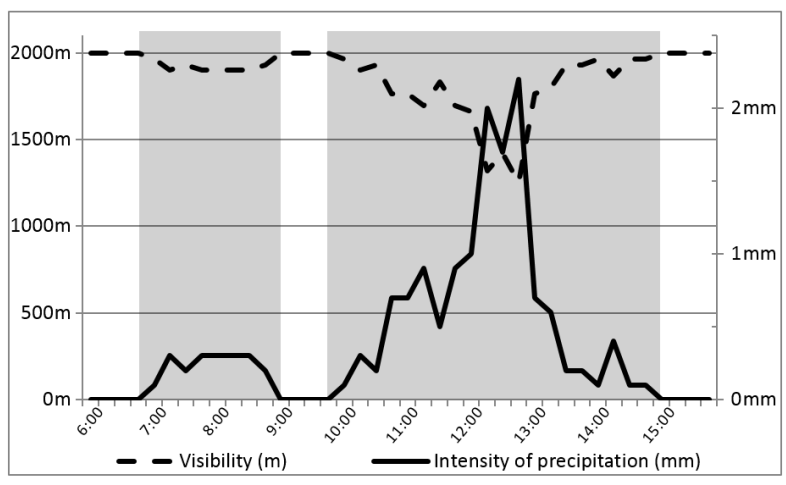

(a) Some variables and the presence of snow

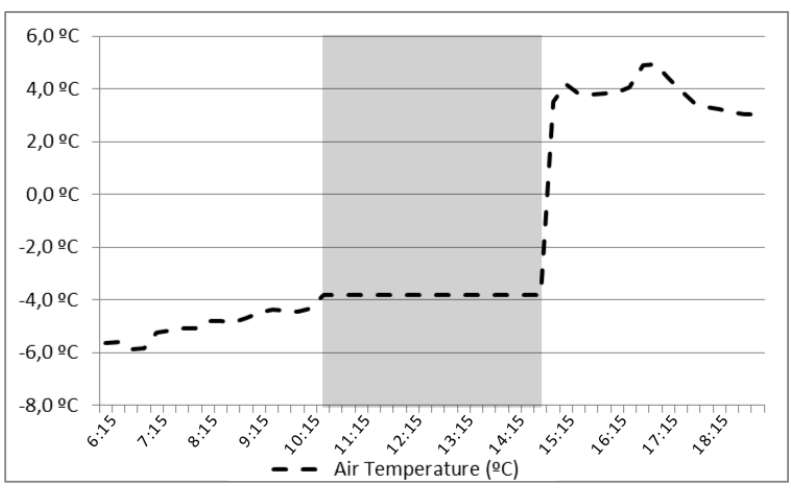

(b) Possible problems in the Air Temperature sensor

Fig. 3. Meteorological variables' different situations example

road operator could, in real time or off-time, assess the system detection and forecasting. This situation is very important because the historic values are based on this assessment.

\section{REAL CASE: SPANiSH A6 MOTORWAY WEATHER PREDICTION}

The presented model has been evaluated using the A6 motorway in Spain. There are 44 meteorological stations located along the entire motorway. This motorway connects the center and North-West of the country and, due to its geographical condition, the presence of ice and snow on the road is quite usual in the winter season.

To study the reliability of the model, four meteorological stations have been chosen in an area with frequent snow and ice on the road. The four stations are located in the A6 motorway at the following kilometric points: kp378 (San Miguel de las Dueñas), kp347 (Manzanal del Puerto), kp324 (Astorga) and kp292 (Valcabado del Pramo).

In order to explain better the whole process in more detail, we shall first show the prediction rules that have been used.

\section{A. Prediction Rules}

The prediction rules are based on the results produced by the Meteosafety and EasyWay projects and the different studies focused on the evolution of adverse weather situations and their impact on road traffic [39].

The former rules in [34] has been updated with a double check: the first condition is set taking into account the results of the Meteosafety and Easyway projects and the second condition is set based on the evolution of the variable with respect to time. Furthermore, the new Certainty value is updated taking this evolution into account.

1) Snow Prediction Rules: The main objective of the Snow Prediction Rules is to give a value of the parameter Certainty in order to detect the presence of snow on the road. When this parameter exceeds the value of $80 \%$, the system is detecting a potential problem and triggers the corresponding alert.

The variables taken into account in the snow prediction rules are the following: Dew occurrence temperature (DewTemp) and its evolution in time ( $\triangle$ DewTemp); Air Relative Humidity (AirHumid); Air Temperature (AirTemp) and its evolution in time ( $\triangle$ AirTemp); Soil surface temperature (SoilSurfTemp) and its evolution in time ( $\Delta$ SoilSurfTemp); Underground temperature (UnderTemp) and its evolution in time ( $\Delta$ UnderTemp); Global Radiation (Radiation) and its evolution in time ( $\triangle$ Radiation); Air Pressure (AirPress) and its evolution in time ( $\triangle$ AirPress); Soil Salinity (Salinity); Intensity of precipitation (PrecipInt) and Soil Freeze Temperature (SoilFreezeTemp).

As an example, the rules described in Rule 1, Rule 2 and Rule 3 show the rules corresponding to the Air Temperature, 
Soil Surface Temperature and Precipitation Intensity variables in the Snow Prediction Rules. The values that have been considered in the rules were empirically obtained during the Meteosafety project development.
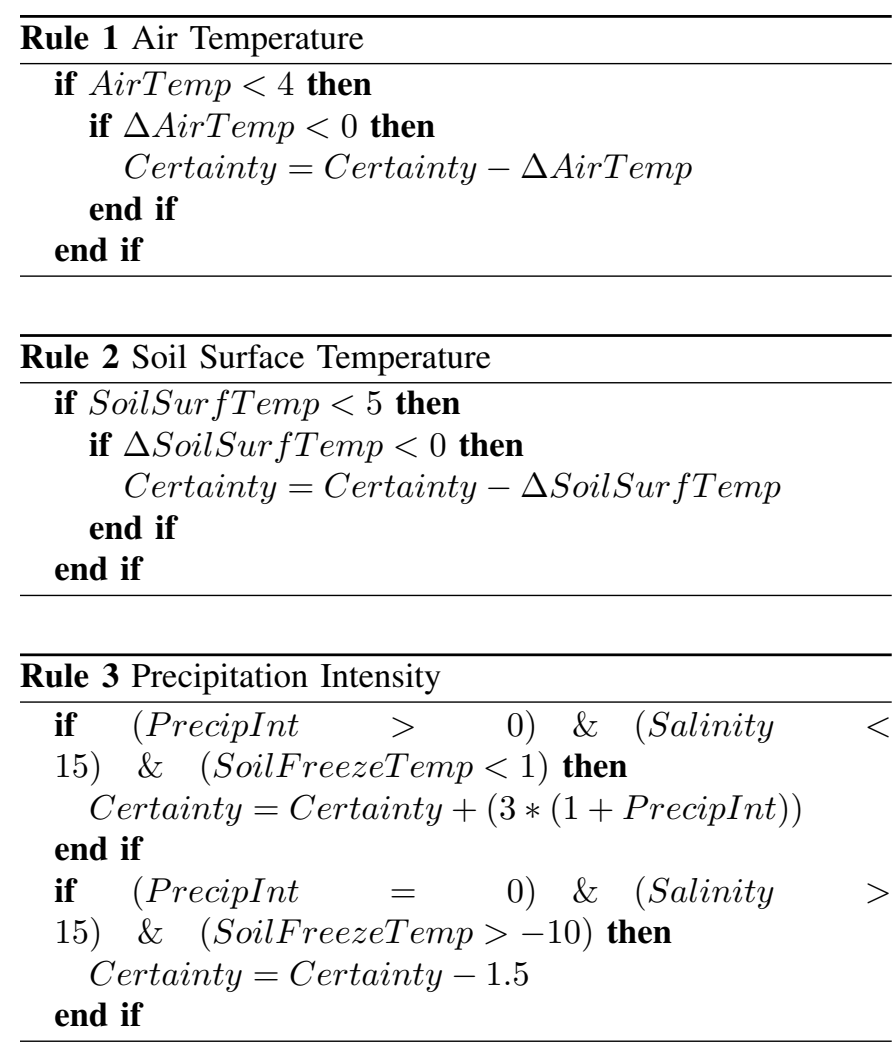

2) Ice Prediction Rules: The main objective of the Ice Prediction Rules is to give a value of the parameter Certainty in order to detect the presence of ice on the road. When this parameter exceeds the value of $80 \%$, the system is detecting a potential problem and triggers the corresponding alert.

The variables taken into account in the ice prediction rules are the following: Air Temperature (AirTemp) and its evolution in time ( $\triangle$ AirTemp); Dew occurrence temperature (DewTemp) and its evolution in time $(\Delta$ DewTemp $)$; Soil Freeze Temperature (SoilFreezeTemp); Soil surface temperature (SoilSurfTemp) and its evolution in time ( $\Delta$ SoilSurfTemp); Underground temperature (UnderTemp) and its evolution in time ( $\Delta$ UnderTemp); Soil Salinity (Salinity); Air Relative Humidity (AirHumid); Global Radiation (Radiation) and its evolution in time ( $\Delta$ Radiation).

As an example, rules described in Rule 4, Rule 5 and Rule 6 show the rules corresponding to the Air Temperature, Soil Surface Temperature and Soil Salinity variables in the Ice Prediction Rules. The values that have been considered in the rules are empirically obtained during the Meteosafety project development.

\section{B. Results and discussion}

As we have seen above, both the ice model and the snow model consider different variables and different limits to make the predictions. In this section, we will see how they work in a real environment and their success in predicting adverse weather conditions.
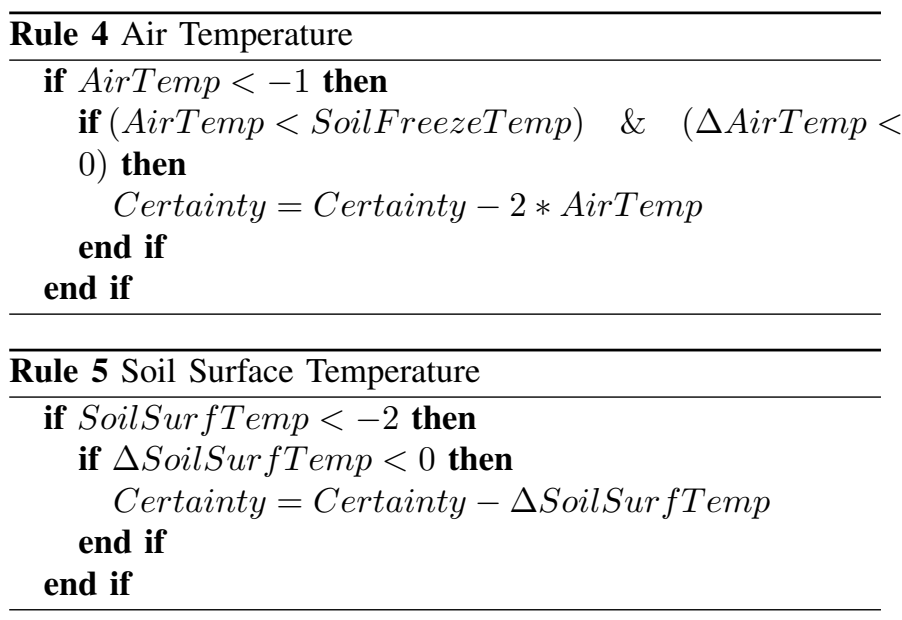

\begin{tabular}{l}
\hline Rule 6 Soil Salinity \\
\hline if Salinity $>2$ then \\
Certainty $=$ Certainty - Salinity $/ 10$ \\
end if
\end{tabular}

1) A simple case: As a first attempt to prove our system, we are going to consider a single station (Station 1) and a brief period of time. We want to compare the outputs of our system with the real situations that occurred and that can be obtained from AEMET (Spanish Metheorological Agency) [40].

Figures 4(a) and 4(b) show an example of some variables measured during 8 hours in Station 1. The figures show how some of these variables behave and show the output of the new prediction model in that space of time. In particular, we show the evolution of three variables; Air Temperature, Soil Surface Temperature and Salinity for the case of Ice Model and Air Temperature, Soil Surface Temperature and Precipitation Intensity for the case of Snow Model. The prediction of ice and snow made by the model, appears as an overlapping shaded area (in light grey) and the real presence of ice and snow are shown in the dark grey shaded area.

The ice model and the snow model consider different variables and different limit values for predictions. In the example shown in Fig 4(b), the air temperature and the soil surface temperature are also very low but not as much as in the ice model. The presence of precipitation, with low temperatures is a sufficient condition for the system to make a prediction of the presence of snow on the road.

In the ice prediction model shown in Figure 4(a), we can see that the values of air temperature and soil surface temperature are very low throughout the period shown (bellow $0^{\circ} \mathrm{C}$ ). However, it can be clearly seen that the presence of salt on the road, takes away the presence of ice in it.

2) Extending the time range: The examples shown in Figure 4 are for a specific weather station and during a very short period of time (12 hours). If we want to study the reliability of the model, as mentioned at the beginning of the section, we have to choose a wider range of stations and time length. To do this, four weather stations were chosen in a period of eight months during the winter season. This is defined as Time Extended Analysis (TEA).

As we have done in Section V-B1 we want to compare 


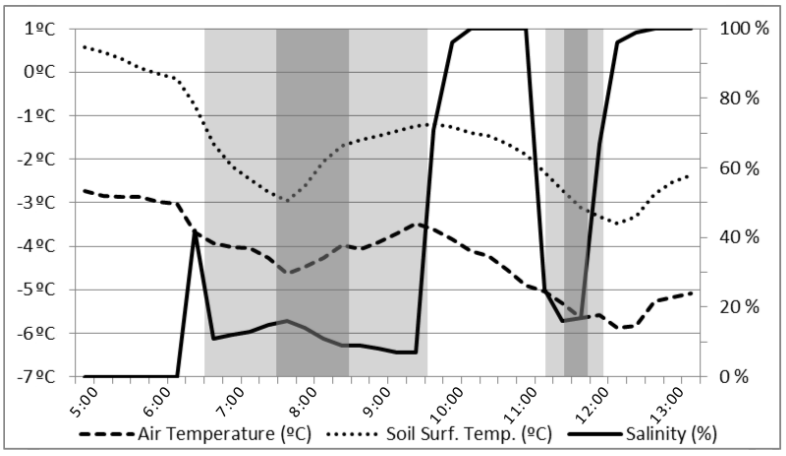

(a) Some variables and the presence of ice

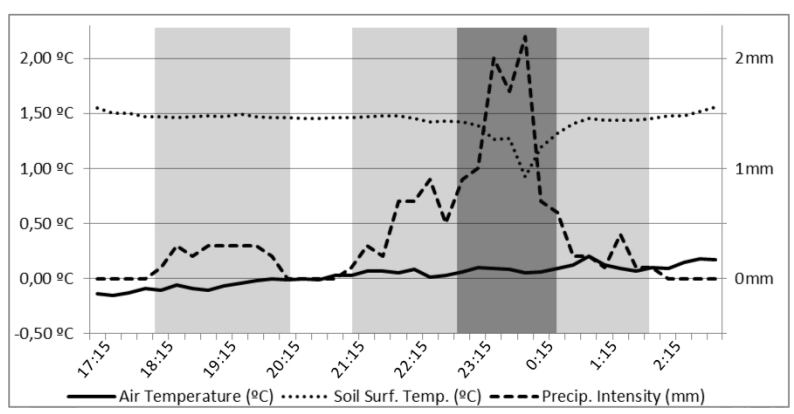

(b) Some variables and the presence of snow

Fig. 4. Relationship between different variables and the presence of ice and snow. Model prediction in shade blue and real presence in shaded red.

the output of our system with the real meteorological events obtained from AEMET. We want to compare the certainty value obtained by our model taking into account the collected data from weather stations and the HIRLAM system and the real presence of ice or snow on the road in the period studied.

In predictive analytics, a confusion matrix (sometimes also called error table or contingency table [41], [42]), contains information about actual and predicted classifications done by a classification system. Two-class classification involves the task of classifying cases into two set of classes with classes 1 (yes) and 0 (no) and with four possible combinations of either true positive (TP), true negative (TN), false positive (FP) or false negative (FN). A false positive is when the outcome is incorrectly classified as yes (or positive), when it is in fact no (or negative). A false negative is when the outcome is incorrectly classified as negative when it is in fact positive. True positives and true negatives are obviously correct classifications.

With data obtained from the output of the model in the TEA and the real data obtained from HIRLAM, two confusion matrices have been built (see Figures 5 and 6). These matrices represent the percentages of success or failure of a classification (in this case, our prediction system). In our study, false positives correspond to the prediction that there is going to be ice or snow and this condition does not occur. On the other hand, the false negative corresponds to when the system does not send an alert and there really is ice or snow on the road.

Another factor to determine the reliability of our predictive method is the accuracy (AC), the sensitivity (S) and the Error
Rate (ER). A good classification method must be sensitive and accurate as much as possible [43]. The AC is the proportion of the total number of predictions that were correct, the $\mathrm{S}$ measures the proportion of positives that are correctly identified as such and the ER the proportion of the wrong predictions. It is determined using the equations:

$$
A C=\frac{a+d}{a+b+c+d}
$$

and

$$
S=\frac{d}{b+d}
$$

where

\begin{tabular}{|c|c|c|c|}
\cline { 3 - 4 } \multicolumn{1}{c|}{} & \multicolumn{2}{c|}{ Real } \\
\hline \multirow{4}{*}{ Predicted } & & Negative & Positive \\
\cline { 2 - 4 } & Positive & b & d \\
\cline { 2 - 4 } & Negative & a & c \\
\hline
\end{tabular}

and th TP,TN,FP and FN rate are obtained with:

$$
\begin{array}{ll}
T P=\frac{d}{c+d} ; & F P=\frac{b}{a+b} ; \\
T N=\frac{a}{a+b} ; & F N=\frac{c}{c+d}
\end{array}
$$
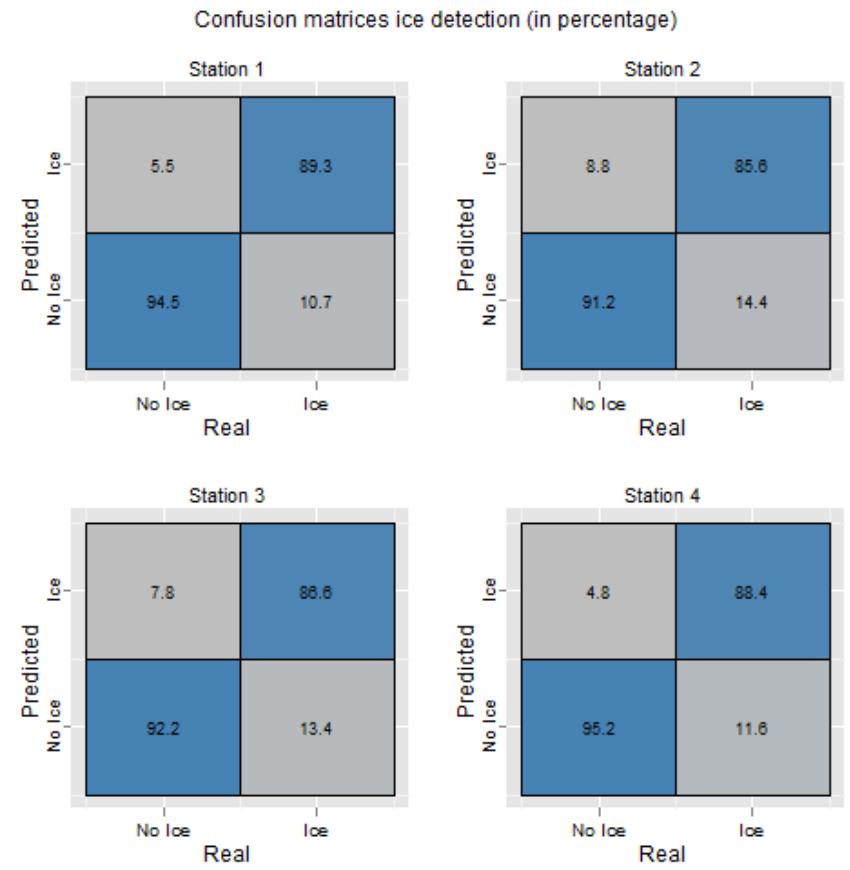

Fig. 5. Confusion matrix for the ice prediction model

With the data obtained and showed in Figures 5 and 6 our system obtains the results shown in Table II.

Table II shows an average AC of $90 \%$ and an average S of $93 \%$ in all the situations and thus an average of $10 \%$ of ER. These results show that the new prediction system we have developed has a high level of accuracy and therefore is a reliable method to inform the users about adverse weather situations in an efficient way. It must be taken into account 


\begin{tabular}{|c|c|c|c|c|c|c|c|c|}
\hline & & $\mathrm{TP}$ & FP & $\mathrm{TN}$ & FN & $\mathrm{AC}$ & $\mathrm{S}$ & ER \\
\hline \multirow{4}{*}{ 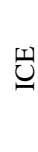 } & Station 1 & 89.3 & 5.5 & 94.5 & 10.7 & 91.9 & 94.2 & 8.1 \\
\hline & Station 2 & 85.6 & 8.8 & 91.2 & 14.4 & 88.4 & 90.7 & 11.6 \\
\hline & Station 3 & 86.6 & 7.8 & 92.2 & 13.4 & 89.4 & 91.7 & 10.6 \\
\hline & Station 4 & 88.4 & 4.8 & 95.2 & 11.6 & 91.8 & 94.9 & 8.2 \\
\hline \multirow{4}{*}{$\begin{array}{l}z \\
z \\
z \\
\text { z }\end{array}$} & Station 1 & 87.2 & 6.9 & 93.1 & 12.8 & 90.15 & 92.7 & 9.85 \\
\hline & Station 2 & 85.5 & 5.5 & 94.5 & 14.5 & 90 & 94 & 10 \\
\hline & Station 3 & 89.4 & 7.2 & 92.8 & 10.6 & 91.1 & 92.5 & 8.9 \\
\hline & Station 4 & 86.1 & 8.6 & 91.4 & 13.9 & 88.75 & 90.9 & 11.25 \\
\hline
\end{tabular}

TABLE II

ICE AND SNOW TP,TN,FP AND FN RATES, AC, S AND ER OF THE 4 STATIONS CONSIDERED
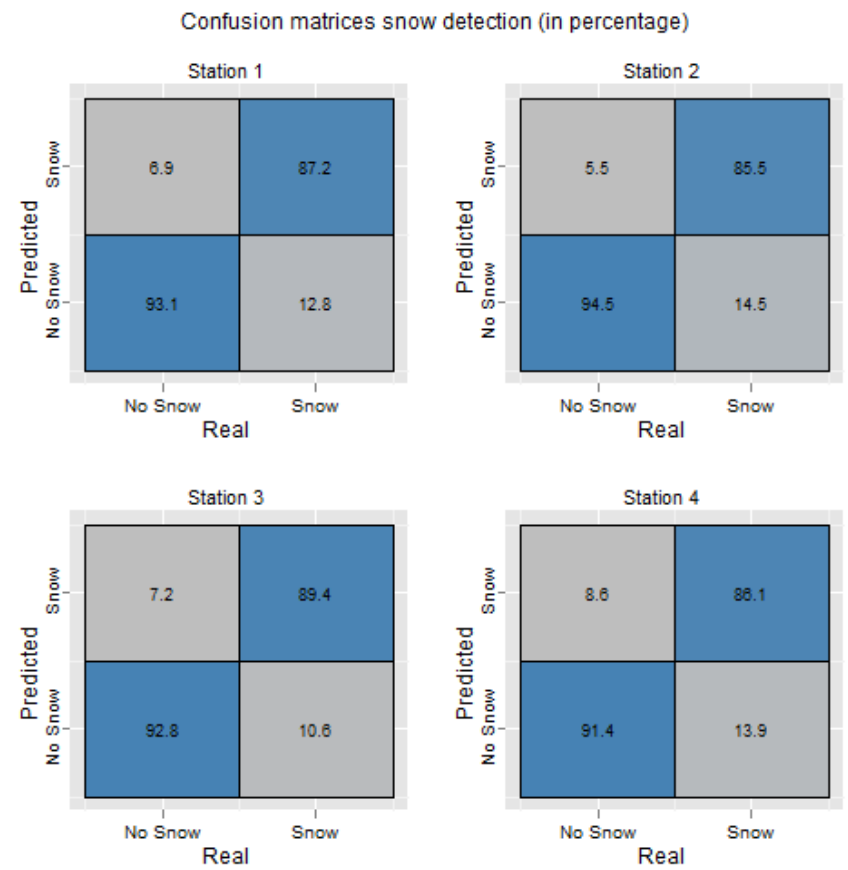

Fig. 6. Confusion matrix for the snow prediction model

that these results are based on predictions made by a nonsupervised system. In case of detection of an extreme weather situation where the drivers may be endangered, the alerts can be supervised by a TCC operator.

\section{CONCLUSIONS AND FUTURE WORK}

This paper presents a new autonomous system to forecast weather conditions in a short time and to give users the information obtained. The system uses a set of algorithms and rules to determine the weather and to forecast dangerous situations in the road network. It has been implemented using a multi-agent approach and tested with real data.

The first test was a study considering a single station and a short time period (12 hours). The results in this case were very good and the study was extended to forecast short-term ( 2 hours) weather taking into account real data in a wider period of time ( 8 months). This second analysis has provided some results with an accuracy of $90 \%$ for short-term weather prediction.
The improvement of the short-term prediction achieved in the work presented in this paper increase the system timeliness (time to detect the incident) and the service provision because no operator is required to warn end-users. We have obtained a very reliable and unsupervised system.

The work to follow this one will be to update the algorithms to take into account information from various stations and the calculations and predictions will be done in a coordinated manner.

\section{REFERENCES}

[1] U.S. Department of Transport, "How do weather events impact roads?" http://www.ops.fhwa.dot.gov/weather/q1_roadimpact.htm, 07 2011, [Online; accessed January 8, 2015].

[2] Dirección General de Tráfico, "Anuario estadstico de accidentes 2013." http://www.dgt.es/Galerias/ seguridad-vial/estadisticas-e-indicadores/publicaciones/ anuario-estadistico-de-accidentes/anuario-accidentes-2013.pdf, Dirección general de Tráfico, Tech. Rep., 2013.

[3] R. Oorni, S. Innamaa, and R. Kulmala, "Road weather service in finland: The impact of error probability on service benefits," Advances in Intelligent and Soft Computing, vol. 81, pp. 187-201, 2010.

[4] H. Alhassan and J. Edigbe, "Influence of rainfall intensity on traffic flow rates modelling techniques," in Proceedings 3rd International Graduate Conference on Science, Engineering and Humanities, 2010.

[5] H. Alhasan and J. Edigbe, "Highway capacity prediction in adverse weather," Journal of Applied Sciences, vol. 11, pp. 2193-2199, 2011.

[6] E. Chung, O. Ohtani, H. Warita, M. Kuwahara, and H. Morita, "Does weather affect highway capacity?" in 5th International Symposium on Highway Capacity and Quality of Service, 2006.

[7] R. Jiang, E. Chung, and J. Lee, "Variable speed limits: Conceptual design for queensland practice," in Australasian Transport Research Forum 2011 Proceedings, 2011.

[8] M. Karkowski and E. Chung, "Intro - intelligent roads, integration of weather effects for traffic indicators forecasting," 2007.

[9] P. Rama and R. Kulmala, "Effects of variable message signs for slippery road conditions on driving speed and headways," Transportation Research Part F: Traffic Psychology and Behaviour, vol. 3, no. 2, pp. $85-94,2000$.

[10] R. Reel, M. Demidovich, J. Rosen, J. Kreideweis, J. Avis, D. Gardne, R. Viste, and M. Hallenbeck, Traffic Data quality Measurement. Federal Highway Administration U.S. Department of Transportation, 2004.

[11] "COOPERS project. co-operative networks for intelligent road safety," 2010. [Online]. Available: http://www.coopers-ip.eu/

[12] "EASYWAY project." 2009-2013. [Online]. Available: http://www. easyway-its.eu

[13] "QUANTIS Project. Quality assessment and assurance methodology for traffic data and information services," 2009. [Online]. Available: http://www.quantis-project.eu

[14] "EIP Project. European ITS Platform," 2014-2015.

[15] E. Takle, "Bridge and roadway frost: Ocurrence and prediction by use of an expert system," Journal of Applied Meteorology, vol. 29, no. 8, pp. 727-734, 1990.

[16] E. S. Takle and P. C. Thomson, "Use of expert systems for roadway weather maintenance decisions," in Semisequicentennial Transportation Conference Proceedings, 1996. 
[17] R. F. Chevalier, G. Hoogenboom, R. W. McClendon, and J. O. Paz, "A web-based fuzzy expert system for frost warnings in horticultural crops," Environmental Modelling \& Software, vol. 35, pp. 84 - 91, 2012.

[18] A. IF, "Impact of road surface temperature and condition on the risk of winter vehicle collisions," Ph.D. dissertation, Carleton University Ottawa, 2005.

[19] Y. Siril, K. Askar, and M. Dougherty, "Expert system to calculate the coefficient of friction- an approach to enhance traffic safety," in Proceedings of the 2004 IEEE conference on Cibernetics and Intelligent Systems, 2004

[20] J. E. Thornes, G. Cavan, and L. Chapman, "Xrwis: the use of geomatics to predict winter road surface temperatures in poland." Meteorological Applications, vol. 12, pp. 83-90, 2005.

[21] P. Undin, L. Rontu, H. Jarvinen, P. Lynch, and J. Calvo, HIRLAM-5 Scientific Documentation. Norrkiping: Swedish Meteorological and Hydrological Institute, 2002.

[22] "HIRLAM Project." visited in 2015. [Online]. Available: http: //www.hirlam.org

[23] B. H. Sass and X. Yang, "A verification score for high resolution nwp: Idealized and preoperational tests," HIRLAM Tecnical report., vol. 69, 2012.

[24] K. Eerola, "Twenty-one years of verification from the hirlam nwp system," Weather and Forecasting journal, vol. 28, no. 1, 2012.

[25] P. Kalina and J. Vokrinek, "Parallel solver for vehicle routing and pickup and delivery problems with time windows based on agent negotiation," in Systems, Man, and Cybernetics (SMC), 2012 IEEE International Conference on, 2012, pp. 1558-1563.

[26] M. Gath, O. Herzog, and S. Edelkamp, "Autonomous and flexible multiagent systems enhance transport logistics," in Emerging Technologies for a Smarter World (CEWIT), 2014 11th International Conference \& Expo on. IEEE, 2014, pp. 1-6.

[27] E. Belyi, I. Patel, A. Reddy, and V. Mago, "A multi-agent based system for route planning," in Human Interface and the Management of Information. Information and Knowledge Design. Springer, 2015, pp. 500-512.

[28] R. Lee and J. Liu, "JADE WeatherMAN: a weather forecasting system using intelligent multiagent-based fuzzy neuro network," Systems, Man, and Cybernetics, Part C: Applications and Reviews, IEEE Transactions onl, vol. 34, no. 3, pp. 369-377, 2004.

[29] S. Cheng, A. Raja, and L. Victor, "Multiagent Meta-level Control for Radar Coordination," Web Intelligence and Agent Systems: An International Journal, vol. 11, no. 2, pp. 81-105, 2013. [Online]. Available: http://mas.cs.umass.edu/paper/512

[30] R. Tornero, J. Martínez, and J. Castelló, “Computing real-time dynamic origin/destination matrices from vehicle-to-infrastructure messages using a multi-agent system," in Highlights on Practical Applications of Agents and Multi-Agent Systems, ser. Advances in Intelligent and Soft Computing. Springer Berlin Heidelberg, 2012, vol. 156, pp. 147-154.

[31] C. Di Napoli, D. Di Nocera, and S. Rossi, "Negotiating parking spaces in smart cities," in Proceeding of the 8th International Workshop on Agents in Traffic and Transportation, in conjunction with AAMAS, 2014.

[32] J. Ma, B. L. Smith, and X. Zhou, "Personalized real-time traffic information provision: Agent-based optimization model and solution framework," Transportation Research Part C: Emerging Technologies, 2015.

[33] T. Shirai, Y. Konaka, J. Yano, S. Nishimura, K. Kagawa, T. Morita, M. Numao, and S. Kurihara, "Multi-agent traffic light control framework based on direct and indirect coordination," in Proceedings of the 7th International Workshop on Agents in Traffic and Transportation, 2012.

[34] I. Martí, V. R. Tomás, L. A. García, and J. J. Martínez, "A multi-agent system for managing adverse weather situations on the road network," Integr. Comput.-Aided Eng., vol. 17, pp. 145-155, April 2010.

[35] "DATEX II. Data exchange specifications for traffic management and information. cen/ts 16157." [Online]. Available: www.datex2.eu

[36] Meteosafety, "Meteosafety. study for the reduction of accidents in adverse weather conditions," http://www.transport-research.info/web/ projects/project_details.cfm?id=38933, 2008-2010, [Online; accessed January 8, 2015].

[37] Multi-agents system standard specifications. [Online]. Available: http://www.fipa.org/specifications

[38] S. Poslad, "Specifying protocols for multi-agent systems interaction," ACM Trans. Auton. Adapt. Syst., vol. 2, no. 4, 2007.

[39] M. Rodriguez-Jara, "Traffic management plan in snowfall situations," in 1st International Seminar on Risk Management for Roads and international workshop on Tsunami. Vietnam, 2006.

[40] AEMET, "Aemet. spanish metheorological agency," http://www.aemet. es/en/portada, [Online; accessed January 13, 2015].
[41] R. Kohavi and F. Provost, "On applied research in machine learning," Editorial for the Special Issue on Applications of Machine Learning and the Knowledge Discovery Process, Columbia University, New York, vol. 30, 1998.

[42] V. M. Patro and M. Patra, "A novel approach to compute confusion matrix for classification of n-class attributes with feature selection," Transactions on Machine Learning and Artificial Intelligence, vol. 3, no. 2, 2015.

[43] F. Gorunescu, Data Mining: Concepts, models and techniques. Springer Science \& Business Media, 2011, vol. 12.

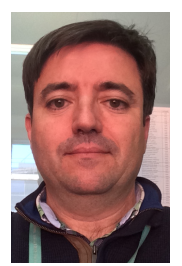

Vicente R. Tomás He received his M.Sc. in Computer Science from the Polytechnic University of Valencia and his PhD in Computer Science from the University of Valencia. In September 2002 he started as an assistant professor at the Universitat Jaume I. $\mathrm{He}$ is currently Associate Professor. He is member of the research group Applying Intelligent Agents. He has participated in numerous research projects related to intelligent transport systems and multiagent systems. His main research areas include: multiagent systems. intelligent transport systems, auto-negotiation and

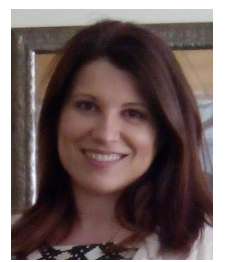

Marta Pla-Castells She received her M.Sc. in Maths from the Universidad de Valencia and the Ph.D degree from Universidad de Valencia, Valencia, Spain, in 2000 and 2009, respectively. She is currently a Senior Researcher at LISITT laboratory at the Institute IRTIC and Assistant Professor at University of Valencia. She has been working on research tasks in Physical Simulation and ITS applications projects since 2000 until the date, being her main background on software engineering and project management.

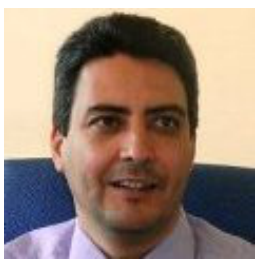

Juan J. Martínez $\mathrm{He}$ is currently an Associate Professor at the School of Engineering, carrying out his research at LISITT in IRTIC. He has been working on ITS applications projects since 1990 until the date, being his main background on traffic and traveler information, software engineering and project management. He has participated in several EU projects from the beginning of his research work and he is the co-author of more than 50 research publications.

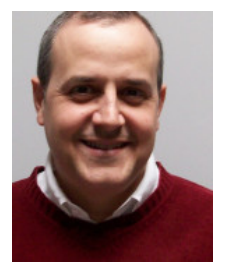

Javier Martínez He is currently an Associate Professor at the School of Engineering, carrying out his research at LISITT in IRTIC. He has been working on ITS applications projects since 1992 until the date, being his main background on traffic and traveler information, ITS applied to tunnel safety and BT sensors applied transport. He has participated in several EU projects from the beginning of his research work and he is the co-author of more than 30 research publications. 